\title{
On rings with trivial torsion parts
}

\section{Bican, P. Jambor, T. Kepka, P. Němec}

\begin{abstract}
In this paper, we exhibit the necessary and sufficient conditions for a ring $R$ to have only the trivial torsion parts with respect to any (hereditary) radical on the category of left $R$-modules.
\end{abstract}

\section{Introduction}

Let $R$ be a ring with identity and $r$ be a (hereditary) radical on the category $R^{\text {mod }}$ of the left $R$-modules, that is, $r$ is an idempotent subfunctor of identity such that $r(M / r(M))=0$ for every $M \in R^{\bmod }$ (in addition, $r$ is left exact). In investigations of radical structure on modules, we often need the condition $r(R)=0$. So it is natural and of interest to study rings having this property for all non-trivial radicals. We shall say that $R$ is a left $R$-ring (T-ring) if $r(R)=0$ for every non-trivial (hereditary) radical $r$ on $R^{\bmod }$.

In this paper, we exhibit the necessary and sufficient conditions for a ring to be either an $R$-ring or a T-ring, supplied with interesting counterexamples. The main result of Section 2 is: $R$ is an $R$-ring (T-ring) iff $R_{n}$ is an $R$-ring (T-ring) for every $n \geq 1$. Section 3 applies the ideas of Gardner's work [1] to an extent of a structural investigation of $T$ and $R$-rings with non-zero socles. Throughout this paper, unless otherwise specified, $R$ stands for a ring with identity and either $T$ or $R$-rings are considered as the left $T$ or $R$-rings. Let us recall ([4]) that the existence of a radical $r$ on $R^{\bmod }$ is equivalent to the existence of a torsion theory $(M, L)$ where 


$$
M=\left\{M \in R_{R}^{\bmod } \mid r(M)=M\right\}=L^{+}=\left\{M \in R^{\bmod } \mid \operatorname{hom}_{R}(M, L)=0, \forall L \in L\right\}
$$

and

$$
L=\left\{M \in R_{R}^{\bmod } \mid \boldsymbol{r}(M)=0\right\}=M^{*}=\left\{L \in R^{\bmod } \mid \operatorname{hom}_{R}(M, L)=0, \forall M \in M\right\} .
$$

In particular ([9]), in the case of a hereditary radical it is equivalent to the existence of a radical filter $E \subseteq Z(R)$, where $Z(R)$ is the set of left ideals of $R$; that is,

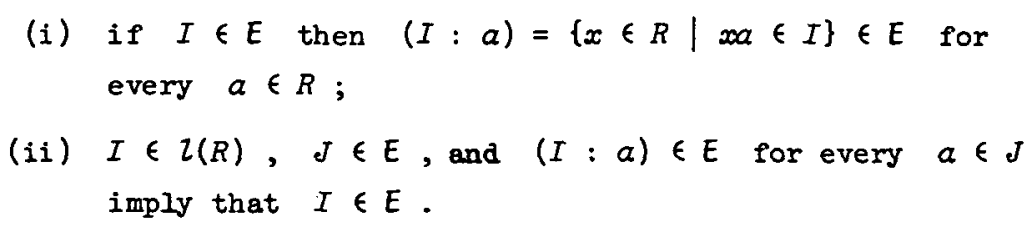

It is essential to know that if $E$ is a radical filter then the corresponding radical $r$ is defined by $r(M)=\{m \in M \mid(0: m) \in E\}$ for every $M \in R^{\bmod }$ and $I \in E$ iff $r(R / I)=R / I$ ([9]). It is easy to see that our definition of a radical filter is equivalent to that of [9]. Note that if $r$ is a radical on $R^{\text {mod }}$ then $r(R)$ is a two-sided ideal since $r$ is a subfunctor of identity and the right multiplication on $R$ is a left $R$-homomorphism. It should be remarked that, obviously, simple rings are $R$-rings and integral domains are T-rings.

We shall frequently use the following notation:

$M \subseteq R$ is right $T$-nilpotent if $\forall\left(a_{1}, a_{2}, \ldots \in M\right) \quad \exists(n \geq 1)$ such that $a_{n} a_{n-1} \cdots a_{1}=0$;

$R$ is a comutative primary ring if the prime radical is a prime ideal;

$I \in Z(R)$ is an essential ideal if $I \neq 0$ and $I \cap J \neq 0$ for every $J \in Z(R), J \neq 0$;

$R_{R}=\left\{M \in R^{\bmod } \mid r(M)=0\right.$ or $r(M)=M$ for every radical $r$ on $\left.R^{\bmod }\right\}$, 


$$
\begin{aligned}
T_{R}=\left\{M \in{ }_{R}^{\bmod } \mid r(M)=0 \text { or } r(M)=M\right. & \text { for every hereditary } \\
& \text { radical } \left.r \text { on } R^{\bmod }\right\} ;
\end{aligned}
$$

$C(R)$ - the center of $R$;

$\hat{M} \quad$ - the injective hull of $M \in R_{R}^{\bmod }$;

$R(+)$ - the underlying abelian group of $R$;

$J(R)$ - Jacobson radical of $R$;

$R^{n} \quad$ - direct product of $n$ copies of $R$;

$R_{n} \quad$ - the full ring of matrices of degree $n$ over $R$.

The scalar matrix corresponding to an element $x \in R$ is the diagonal matrix with all the elements on the diagonal equal to $x$.

For simplicity, by $M \in T_{R}$ or $T_{R}^{h}$ or $F_{R}$ or $F_{R}^{h}$ we mean that $M$ is a torsion class, hereditary torsion class, torsion-free class and hereditary torsion-free class respectively.

\section{On $T$ and $R$-rings}

THEOREM 1.1. Let $R$ be a ring and $M \subseteq R$ be a subset. Then $E_{M}=\left\{I \in Z(R) \mid \forall\left(a_{1}, a_{2}, \ldots \in M\right) \forall(s \in R) \exists(n \geq 1)\left(a_{n} a_{n-1} \ldots a_{1} s \in I\right)\right\}$ is a radical filter and

(i) if $M$ is a left ideal then $E_{M}$ is contained in the least radical filter containing $M$,

(ii) if $M$ is a two-sided ideal then $E_{M}$ is the least radical filter containing $M$,

(iii) $E_{M}=2(R)$ iff $M$ is right T-nilpotent.

Proof. Let $I \in E_{M}, t \in R$ and suppose that $a_{1}, a_{2}, \ldots \in M$ and $s \in R$. Then there is $n \geq 1$ such that $a_{n} a_{n-1} \ldots a_{1} s t \in I$, that is, . $a_{n} a_{n-1} \cdots a_{1} s \in(I: t)$ and consequently $(I: t) \in E_{M}$. If $K$ is a left ideal such that for every $k \in I,(K: k) \in E_{M}$, then there is 
$n \geq 1$ such that $a_{n} a_{n-1} \cdots a_{1} s=u \in I$ and $(K: u) \in E_{M}$. Hence, there is $m \geq 1$ such that $a_{n+m} \cdots a_{n+1} a_{n} \ldots a_{1} s \in K$.

(i) Let $K \in E_{M} \backslash C$ where $C$ is the least radical filter containing $M$. By the definition of radical filter there is $a_{1} \in M$ such that $\left(K: a_{1}\right) \vDash C$ and consequently there is a sequence $a_{1}, a_{2}, \ldots \in M$ such that $\left(\left(\ldots\left(\left(k: a_{1}\right): a_{2}\right): \ldots\right): a_{n}\right)=\left(k: a_{n} a_{n-1} \ldots a_{1}\right) \notin c$ for every $n \geq 1$, which yields a contradiction with the definition of $E_{M}$.

(ii) If $M$ is a two-sided ideal then obviously $M \in E_{M}$.

(iii) It is easy to show that $E_{M}=Z(R)$ iff $0 \in E_{M}$.

COROLLARY 1.2. If $R$ is a commutative ring, $I$ is an ideal in $R$ and $E_{I}^{\prime}=\{K \in Z(R) \mid K \subseteq I$ and $I / K$ is $T$-nilpotent in $R / K\}$, then $E_{I}=\left\{J \in Z(R) \mid \exists\left(K \in E_{I}^{\prime}\right)(K \subseteq J)\right\}$ is the least radical filter containing $I$.

THEOREM 1.3. Let $R$ be a ring. If $(0: a)$ is right T-nilpotent for every $a \in R, a \neq 0$ then $R$ is a T-ring. Conversely, if $R$ is a T-ring then (0:Ra) is right $T$-nilpotent for every $a \in R, a \neq 0$.

Proof. The sufficient condition follows right from Theorem 1.1. For the necessary condition, since $(0: R a)$ is a two-sided ideal, $E_{(0: R a)}$ is the least radical filter containing $(0: R a)$ by Theorem 1.1 (ii). If $a \neq 0$ then $(0: R a) \subseteq(0: a) \in E_{(0: R a)}=Z(R)$, since $R$ is a $T$-ring; and Theorem 1.1 ( $i$ ii) finishes the proof.

COROLLARY 1.4. Let $R$ be a commutative ring. Then $R$ is a T-ring iff $(0: a)$ is T-nilpotent for every $a \in R, a \neq 0$.

COROLLARY 1.5. Every commutative T-ming is primary.

PROPOSITION 1.6. Let $R$ be a r-ring and $e \in R$ be a central idempotent. Then $e=0$ or $e=1$.

Proof. Put $K=e R$. Then $K^{2}=K$ and $K$ is a two-sided ideal. If $(0: e)=0$ then, obviously, $e=1$. Suppose that $a \in(0: e)$, 
$a \neq 0$. Then $K \subseteq(0: a) \in E$ where $E=\{I \in Z(R) \mid K \subseteq I\}$ is a radical filter containing $K$ (it needs just a tedious checking of the radical filter's properties). Since $R$ is a T-ring, $0 \in E$ and consequently $K=0$.

REMARK 1.7. (i) By Proposition 1.6, no direct product of 2 rings is a $T$-ring and consequently $T$-rings are not closed under quotient rings (for example, consider the ring of integers).

(ii) By Corollary 1.4, the commutative T-rings are closed under the subrings containing the identity. On the other hand, generally it is not so in the non-commutative case. For, consider the full matrix ring of degree $n>1$ over a field. It is an R-ring which contains an idempotent $e$ different from zero and identity and the subring generated by $e$ and 1 is not a T-ring.

PROPOSITION 1.8. Let $R$ be a T-ring, $0 \neq a \in C(R)$ and $(0: a) \neq 0$. Then

(i) if $0 \neq M \in{ }_{R} \bmod$ then there is $m \in M, m \neq 0$, such that $a \in(0: m)$,

(ii) (0:a) is an essential left ideal of $R$,

(iii) (0:a) is right T-nilpotent,

(iv) a is nilpotent.

Proof. (i) Consider $M_{a}=\left\{M \in{ }_{R}^{\bmod } \mid m \in M, m \neq 0 \Rightarrow a m \neq 0\right\}$. Then $M_{a} \in F_{R}^{h}$. For, it is sufficient to show that $M_{a}$ is closed under the injective hulls. Let $M \in M_{a}$. Since $a \in C(R), D=\{m \in \hat{M} \mid a m=0\}$ is a submodule of $\hat{M}$ and $D \cap M=0$. Hence $D=0$. Now, by the hypothesis $R \vDash M_{a}$ and since $R$ is a T-ring, $M_{a}=0$.

The rest is an easy consequence of (i) and Theorem 1.3.

COROLLARY 1.9. Let $R$ be a T-ring. Then $R(+)$ is either torsionfree or a p-group, for some prime $p$.

PROPOSITION 1.10. Let $R$ be a ring. Then the following are equivalent: 
(i) $R$ is on R-ring;

(ii) if $A, B \in{ }_{R}^{\bmod }$ and $\operatorname{hom}_{R}(A, B)=0$, then either $B=0$ or $\operatorname{hom}_{R}(A, R)=0$;

(iii) for every non-zero left $i$ deal $I$ and every non-zero $M \in R_{R}^{\bmod }, \operatorname{hom}_{R}(I, M) \neq 0$;

(iv) for every non-trivial left ideal $I, \operatorname{hom}_{R}(I, R / I) \neq 0$,

(v) for every non-trivial two-sided ideal I, $\operatorname{hom}_{R}(I, R / I) \neq 0$.

Proof. (i) $\Rightarrow(i i) \Rightarrow(i i i) \Rightarrow(i v) \Rightarrow(v)$ is obvious.

$(v) \Rightarrow(i)$. If $r$ is a radical on $R^{\bmod }$ then $r(R)$ is a two-sided ideal and $\operatorname{hom}_{R}(r(R), R / r(R))=0$. Hence $r(R)=0$ or $r(R)=R$.

PROPOSITION 1.11. Let $R$ be a ring. Then

(i) if $R$ is an $R$-ring then for every non-zero left ideal $I$ and every simple module $M$ there is a left ideal $K$ such that $K \subseteq I$ and $I / K \cong M$,

(ii) if for every non-trivial two-sided ideal $I, I$ is projective and there is a left ideal $S$ such that $I \subseteq S$ and $\operatorname{hom}_{R}(I, R / S) \neq 0$, then $R$ is an $R$-ring.

Proof. (i) It follows straight from Proposition 1.10 (iii).

(ii) We shall prove condition 1.10 (v). Let $I$ be a non-trivial two-sided ideal. Then we have the exact sequence

$$
\operatorname{hom}_{R}(I, R / I) \rightarrow \operatorname{hom}_{R}(I, R / S) \rightarrow \operatorname{ext}_{R}(I, S / I)=0 .
$$

Since $\operatorname{hom}_{R}(I, R / S) \neq 0, \operatorname{hom}_{R}(I, R / I) \neq 0$.

PROPOSITION 1.12. Let $R$ be an R-ming and $I$ a left ideal such that $I R \neq R$. Then for every left ideal $K, I K=K \Rightarrow K=0$.

Proof. Put $A_{I}=\left\{M \in{ }_{R}^{\bmod } \mid I M=M\right\}$. It is easy work to show that $A_{I} \in T_{R}$. Let $K$ be a non-zero left $i$ deal and suppose that $K \in A_{I}$. 
Then $R \in A_{I}$ as well, since $R$ is an $R$-ring, and it yields a contradiction.

PROPOSITION 1.13. Let $R$ be a ring such that for every non-trivial two-sided ideal $I, I^{2} \neq I$. If $M$ is a projective module and $r(M)=M$, for some non-trivial radical $r$, then $M=0$.

Proof. Let $M \neq 0$ be projective and $r(M)=M$ for some non-trivial radical $r$. Consider the least torsion class $M$ containing $M$. Since $M$ is projective, the corresponding torsion-free class $M^{*}$ is a hereditary torsion class which is closed under the direct products, which implies that the corresponding radical filter $E$ is closed under intersections, and consequently $\bigcap_{I \in E} I=K$ is an idempotent two-sided ideal. Hence $K=0$ or $K=R$, a contradiction.

COROLLARY 1.14. Let $R$ be a ring. If every non-trivial two-sided ideal is projective and not idempotent then $R$ is an $R$-ming.

EXAMPLE 1.15. Let $G$ be a subgroup of the additive group of real numbers.such that there exists a sequence $\left\{a_{i}\right\}_{i=1} \subset G \cap(0,1)$ satisfying $\sum_{i=1}^{\infty} a_{i}<1$. Consider the vector space $V$ over a field $F$ having the basis $A=G \cap(0,1)$. We shall define a binary operation * on $A \cup\{\overline{0}\}$, where $\overline{0}$ is the zero element of $V$, by the following manner: if $a, b, a+b \in A$ then $a * b=a+b, a * b=\overline{0}$ otherwise. We can easily extend the operation * onto the whole $V$ and we get an F-algebra. The following statements are valid:

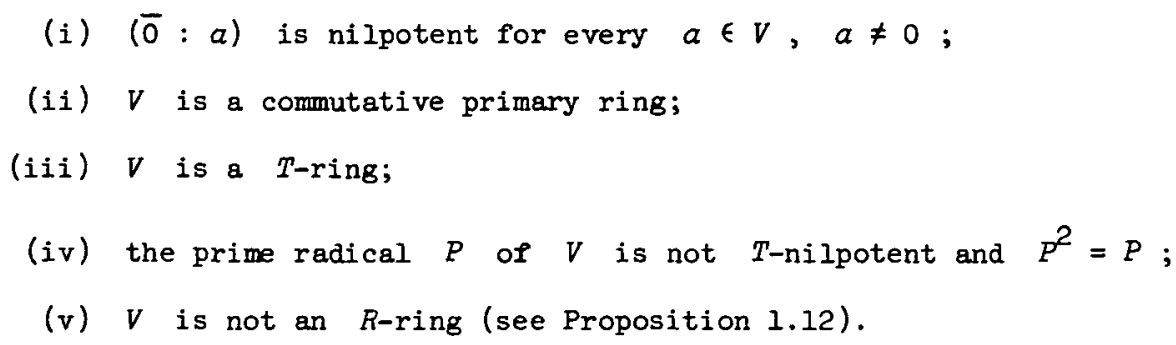


EXAMPLE 1.16. Consider $S=Z \times Q$, where $Z$ is the additive group of integers and $Q$ the additive group of rational numbers. Define the following binary operation on $S$ :

$$
\left(z_{1}, q_{1}\right) *\left(z_{2}, q_{2}\right)=\left(z_{1} z_{2}, z_{1} q_{2}+z_{2} q_{1}\right) \text {. }
$$

Then $S$ becomes a commutative primary ring with prime radical nilpotent of degree 2 . Hence $S$ is a $T$-ring which is not an $R$-ring (see Proposition 1.11). This example is based on the ideas of [5].

\section{Full matrix rings over $T$ and $R$-rings}

DEFINITION 2.1. Let $R$ be a ring, $M \in \frac{\bmod }{R}^{\operatorname{mon}} N$ be a submodule in $M$. We shall say that $N$ satisfies the condition $(T)$ in $M$ if $0 \neq N \neq M$ and there exist $x \in N, y \in M W$ such that $(0: x) \subseteq(N: y)$.

PROPOSITION 2.2. Let $R$ be a ring, $M \in{ }_{R}^{\bmod }$ and $N$ be a submodule in $M$. Then the following are equivalent:

(i) there is a hereditary radical $r$ on $k^{\bmod }$ such that

$$
r(M)=N ;
$$

(ii) $N$ does not satisfy (T) in $M$.

Proof. (i) $\Rightarrow$ (ii). Suppose that $O \neq N \neq M$ and $N$ satisfies (T) in $M$, that is, there is $x \in N$ and $y \in M W$ such that $(0: x) \subseteq(N: y)$. The map $f: R x \rightarrow M / N, a x \mapsto a y+N$ is a well-defined homomorphism and it yields a contradiction, since $r(R x)=R x$ and $r(M / N)=0$.

$(i i) \Rightarrow(i)$. Without loss of generality we can assume that $0 \neq N \neq M$. Consider the least hereditary torsion class $M$ containing $N$ and $r$ be the corresponding hereditary radical. Obviously $N \subseteq r(M)$. If $N \neq r(M)$ then there is a submodule $K \subseteq N$ and a non-zero homomorphism $f: K \rightarrow r(M) / N$. Hence there are $k \in K$ and $y \in r(M) W$ such that $f(k)=y+N$ and consequently $(0: k) \subseteq(N: y)$, a contradiction.

COROLLARY 2.3. Let $R$ be a ring and $M \in{ }_{R}^{\bmod }$. Then the following are equivalent:

(i) $M \in T_{R}$; 
(ii) every non-trivial submodule of $M$ satisfies (T).

THEOREM 2.4. Let $R$ be a ring. Then the following are equivalent:

(i) $R$ is a T-ring;

(ii) every non-trivial left ideal satisfies $(T)$ in $R$;

(iii) every non-trivial two-sided ideal satisfies $(T)$ in $R$.

Proof. (i) $\Rightarrow$ (ii) by Corollary 2.3.

(ii) $\Rightarrow$ (iii) obvious.

(iii) $\Rightarrow$ (i) by Proposition 2.2, considering the fact that any torsion part of $R$ is a two-sided ideal.

THEOREM 2.5. Let $R$ be a ring. Then

(i) if $R$ is a T-ring, then for every $n \geq 1$, the fulz matrix ring $R_{n}$ is a T-ring,

(ii) if there is $n \geq 1$ such that $R_{n}$ is a T-ring then $R$ is a T-ring.

Proof. (i) Let $K$ be a non-trivial two-sided ideal in $R_{n}$. It is easy to see that there is a non-trivial two-sided ideal $I$ in $R$ such that $K=I_{n}$, that is, $K$ is a full matrix ring (possibly without identity) over $I$. According to Theorem 2.4 ( $i$ ii), there are $x \in I$ and $y \in R \backslash$ such that $(0: x) \subseteq(I: y)$. If $X, Y \in R_{n}$ are the corresponding scalar matrices then obviously $X \in K, Y \in R_{n} \backslash K$ and $(0: X) \subseteq(K: Y)$. Now it suffices to use Theorem 2.4 (iii).

(ii) Let $R_{n}$ be a T-ring, for some $n \geq 1$. There is a bifection $f$ between left ideals of $R_{n}$ and $R$-submodules of $R^{n}$ given by $I \mapsto f(I), f(I)$ is a submodule in $R^{n}$ consisting of all the rows of matrices from $I$. If $M$ is a non-trivial submodule of $R^{n}$ then there are matrices $A, B$ such that $A \in f^{-1}(M), B \in R_{n} \backslash f^{-1}(M)$ and $(0: A) \subseteq\left(f^{-1}(M): B\right)$ (see Theorem $2.4(i i)$ ). Since $B \in R_{n} \backslash f^{-1}(M)$, 
there is $1 \leq i \leq n$ such that the $i$-th row of $B$ does not lie in $M$. Put $c \in R_{n}$ as follows: $C=\left(c_{k l}\right), c_{i i}=1$ and $c_{k l}=0$ otherwise. Since $(0: A) \subseteq\left(f^{-1}(M): B\right)$, we get

$$
(0: C A)=((0: A): C) \subseteq\left(\left(f^{-1}(M): B\right): C\right)=\left(f^{-1}(M): C B\right) \text {. }
$$

Let $x$ be the $i$-th row of $C A$ and $y$ be the $i$-th row of $C B$. Obviously $x \in M$ and $y \in R^{n} W$. Consider $a \in(0: x)$ and denote by $D$ the corresponding scalar matrix. Then $D C A=0$, hence $D C B \in f^{-1}(M)$ and consequently ay $\in M$. Now, by Corollary 2.3, $R^{n} \in T_{R}$ and since $T_{R}$ is closed under submodules, $R \in T_{R}$.

PROPOSITION 2.6. Let $R$ be a ring and $N$ be a submodule of an $R$-module $M$. Then the following are equivalent:

(i) there is a radical $r$ on $R^{\bmod }$ such that $r(M)=N$;

(ii) $\operatorname{hom}_{R}(N, M / N)=0$.

Proof. (i) $\Rightarrow$ (ii) is obvious.

$(i i) \Rightarrow(i)$. Let $A$ be the least torsion-free class containing $M / N$ and $r$ be the corresponding radical. Obviously $N \subseteq r(M)$. On the other hand, $\operatorname{hom}_{R}(r(M) / N, M / N)=0$ implies that $r(M) \subseteq N$.

COROLLARY 2.7. Let $M$ be an R-module. Then the following are equivalert:

(i) $M \in R_{R}$,

(ii) if $N$ is a non-trivial submodule of $M$ then $\operatorname{hom}_{R}(N, M / N) \neq 0$.

THEOREM 2.8. Let $R$ be a ring. Then

(i) if $R$ is an R-ring then for every $n \geq 1$, the full matrix ring $R_{n}$ is an $R$-ring,

(ii) if there is $n \geq 1$ such that $R_{n}$ is an $R$-ring then $R$ is an R-ring. 
Proof. (i) Let $K$ be a two-sided ideal in $R_{n}$. Then there is a two-sided ideal $I \subseteq R$ such that $K=I_{n}$, that is, $K$ is the full matrix ring (possibly without identity) over $I$ and if $S=R / I$ then $R_{n} / K \cong S_{n}$ as $R_{n}$-modules. Suppose that $0 \neq K \neq R_{n}$, then $0 \neq I \neq R$ and there is a non-zero $f \in \operatorname{hom}_{R}(I, R / I)$. Hence we can make $f$ into $\bar{f} \in \operatorname{hom}_{R_{n}}\left(K, R_{n} / K\right)$ by $\bar{f}\left(\left(a_{i j}\right)\right)=\left(f\left(a_{i j}\right)\right)$ and $\bar{f} \neq 0$, so that, with respect to Proposition $1.10(v), R_{n}$ is an $R$-ring.

(ii) Let $M$ be a non-trivial $R$-submodule of $R^{n}$ and $I$ be the corresponding left ideal in $R_{n}$. By Proposition 1.10 (iv), there is a non-zero $f \in \operatorname{hom}_{R_{n}}\left(I, R_{n} / I\right)$ and consequently there is $A=\left(a_{i j}\right) \in I$. such that $f\left(\left(a_{i j}\right)\right)=\left(b_{i j}\right)+I \neq I$. Without loss of generality we can assume that the first row of $\left(b_{i j}\right)$ does not lie in $M$. Hence we can make $f$ into non-zero $\bar{f} \in \operatorname{hom}_{R}\left(M, R^{n} / M\right)$ by $\bar{f}(m)=\left(c_{1 j}\right)+M$. , where

$$
f\left(\left(\begin{array}{ccc}
m_{1}, & \ldots, & m_{n} \\
0, & \ldots, & 0 \\
\ldots . & \\
0, & \ldots, & 0
\end{array}\right)\right)=\left(c_{i j}\right)+I,
$$

and an application of Corollary 2.7 shows that $R^{n} \in R_{R}$.

PROPOSITION 2.9. Let $R$ be such a T-ring that every two-sided ideal $I$ is in the form $I=a R=R a$, for some $a \in R$. Then $R$ is an R-ring.

Proof. Suppose that $I$ is a non-trivial two-sided ideal. Then by Theorem 2.4 (iii) there is $x \in I$ and $y \in R \backslash$ such that $(0: x) \subseteq(I: y)$ and since $I=a R, x=a b$ for some $b \in R$. Hence $(0: a) \subseteq(0: x)$ and there is a non-zero $f \in \operatorname{hom}_{R}(I, R / I)$ such that $f(d a)=d y+I$; that is, by Proposition $1.10(v)$, the proof is Pinished.

REMARK 2.10. The authors do not know whether, in general, the polynomial rings over $T$-rings are $T$-rings. However, the following is 
true.

PROPOSITION 2.11. Let $R$ be a commutative T-ring with nilpotent prime radical $P(R)$. Then $R[x]$ is a T-ring.

Proof. Denote by $n$ the degree of nilpotency of $P(R)$. Let $g \in R[x]$ with $(0: g) \neq 0$ and $h \in(0: g)$. It is well known that the coefficients of $h$ are zero divisors in $R$ (see, for example, [1], Chapter 1, exercise 2), and therefore they lie in $P(R)$. Now it is easy to see that $(0: g)$ is nilpotent of degree $n$ and Theorem 1.3 finishes the proof.

\section{On $T$ and $R$-rings with non-zero socles}

THEOREM 3.1. The following conditions for a ring $R$ are equivalent:

(i) $R$ is a left T-ring with non-zero left socle;

(ii) all simple left R-modules are isomorphic and all nonzero left $R$-modules have non-zero socles;

(iii) $R^{\bmod }$ has only two hereditary torsion theories;

(iv) $R$ is isomorphic to a fulz matrix ring over a local ring having left socle sequence;

(v) $J(R)$ is right T-nilpotent and $R / J(R)$ is a simple semisimple artinian ring.

Proof. (i) $\Rightarrow$ (ii) Let $I$ be a minimal left ideal in $R$. By (i), $R$ lies in the least torsion class containing $I$. Therefore $\operatorname{hom}_{R}(I, M) \neq 0$ for every non-zero left $R$-module $M$ and (ii) easily follows.

$(i i) \Rightarrow(i i i)$. See [1], Proposition 2 .

(iii) $\Rightarrow$ (i). Obvious.

(iii) $\Leftrightarrow(i v)$. See [6], Theorem 1 .

(iii) $\Leftrightarrow(v)$. See [1], Theorems 4 and 6 .

THEOREM 3.2. Let $R$ be a ring. Then the following are equivalent:

(i) $R$ is a left $R$-ring with non-zero left socle and $J(R)$ is left T-nilpotent; 
(i') $R$ is a right $R$-ring with non-zero right socle and $J(R)$ is right T-nilpotent;

(ii) $R^{\bmod }$ has only two torsion theories;

(ii') $\bmod _{R}$ has only two torsion theories;

(iii) $J(R)$ is left and right $T$-nilpotent and $R / J(R)$ is a simple semisimple artinian ring;

(iv) $R$ is left and right perfect and has only one simple module up to isomorphism;

(v) $R$ is isomorphic to a full matrix ring over a left and right perfect local ring.

Proof. It clearly suffices to prove the equivalence of the left-hand forms, since condition $(i i i)$ is self-dual.

(i) $\Leftrightarrow$ (ii). It follows from Theorem $3.1(v)$ and [7], Theorem 3 .

(ii) $\Leftrightarrow$ (iii). See [7], Theorems 3 and 6 .

(iii) $\Leftrightarrow$ (iv). See [2], Theorem $P,(1) \Leftrightarrow(2)$.

(iii) $\Leftrightarrow(v)$. See [3], the main theorem, (IA) $\Leftrightarrow$ (IF).

REMARK 3.3. These conditions are equivalent to many others; see, for example, [3], [6].

COROLLARY 3.4. Let $R$ be a commutative ring with non-zero socle. Then $R$ is a T-ming iff it is an R-ring.

PROPOSITION 3.5. Let $R$ be a T-ring with non-zero socte. Then the following are equivalent:

(i) $R$ is an R-ring;

(ii) all submodules of projective modules contain maximal submodules;

(iii) all left ideals contain maximal submodules.

Proof. $(i) \Rightarrow(i i)$. Let $A$ be the least torsion-free class containing all simple $R$-modules. Obviously $R \in A$ and hence every submodule of a projective module has a simple epimorphic image. Thus it contains a maximal submodule. 
(ii) $\Rightarrow$ (iii) is trivial.

$(i i i) \Rightarrow(i)$. By Theorem $3.1(i i)$, every non-zero left $R$-module has a simple submodule unique up to isomorphism, so that ( $i i i$ ) gives $\operatorname{hom}_{R}(I, M) \neq 0$ for every non-zero left ideal $I$ and every non-zero left $R$-module $M$. Now it suffices to use Proposition 1.10 ( $i$ ii).

\section{Weakly dense submodules}

Let $R$ be a ring and $M \in{ }_{R}^{\bmod }$. Then $E(M)$ will be the set consisting of the zero submodule and of all essential submodules of $M$. Further we shall denote by $M_{M}$ the least hereditary torsion class containing $M$ and by $r_{M}$ the corresponding radical.

DEFINITION 4.1. Let $R$ be a ring and $M \in{ }_{R}^{\bmod }$. A submodule $N \subseteq M$ is called weak $l y$ dense in $M$ if there are $K \in E(M)$ and $m \in M \backslash K$ such that for every $n \in M$ and $a \in R \backslash(K: m),(N: n) \notin(K:$ am $)$.

PROPOSITION 4.2. Let $M \in R^{\bmod }$ and $N \subseteq M$ be a submodule. Then $N$ is weakly dense in $M$ iff there are $K \in E(M)$ and $m \in M \backslash K$ such that $\operatorname{hom}_{R}(B / N, R(m+K))=0$ for every submodule $B, N \subseteq B \subseteq M$.

Proof. (i) Let $N$ be weakly dense in $M$ and $K, m$ be as in Definition 4.1. Let $f: B / N \rightarrow R(m+K)$ be a non-zero homomorphism. There is $b \in B$ such that $f(b+N)=a m+K \neq K$. Hence $a \in R \backslash(K: m)$ and $(N: b) \subseteq(k: a m)$, a contradiction.

(ii) If $N$ is not weakly dense in $M$ then for every $K \in E(M)$ and $m \in M \backslash K$ there are $n \in M, a \in R \backslash(K: m)$ such that $(N: n) \subseteq(K: a m)$. Hence $f:(N+R n) / N \rightarrow R(m+K)$ given by $x n+N \mapsto x c m+K$, is a non-zero homomorphism.

PROPOSITION 4.3. Let $M \in R^{\bmod }$ and $N \subseteq M$ be a submodule. If $N$ is not weakly dense in $M$ then $M \in M_{M / N}$.

Proof. Let $m \in M$ be a non-zero element. As $N$ is not weakly dense in $M$, there is $B, N \subseteq B \subseteq M$, such that $\operatorname{hom}_{R}(B / N, R m) \neq 0$. Hence $r_{M / N}(R m) \neq 0$, so that $R m \cap r_{M / N}(M) \neq 0$. Therefore $K=r_{M / N}(M) \in E(M)$. 
Now, from Proposition 4.2, we have $K=M$.

DEFINITION 4.4. Let $M \in R^{\bmod }$ and $N \subseteq M$ be a submodule. Then $N$ is called dense in $M$ if $r_{M / N}(M)=0$, that is, if $\operatorname{hom}_{R}(B / N, M)=0$ for all $B, N \subseteq B \subseteq M$.

PROPOSITION 4.5. Let $M \in{ }_{R}^{\bmod }$ and $N \subseteq M$ be a submodule. Then $N$ is dense in $M$ iff $(N: n) \notin(0: m)$ for alz $m, n \in M, m \neq 0$.

Proof. This is an immediate consequence of Definition 4.4 .

PROPOSITION 4.6. Let $M \in{ }_{R}^{\bmod }$. If $M \in T_{R}$ then every weakly dense submodule in $M$ is dense in $M$.

Proof. It follows from Proposition 4.3 and Definition 4.4 .

THEOREM 4.7. Let $R$ be a ring. Then the following are equivalent:

(i) $R$ is a T-ring;

(ii) every weakly dense left ideal of $R$ is dense in $R$.

Proof. (i) $\Rightarrow(i i)$. See Proposition 4.6 .

$(i i) \Rightarrow(i)$. Let $r$ be a hereditary radical and $E$ the corresponding radical filter. If $E$ contains only dense left ideals then $r(R)=0$. Let $I \in E, I$ be not dense in $R$. Then $I$ is not weakly dense in $R$ and hence $r_{R / I}(R)=R$ by Proposition 4.3. However $r(R / I)=R / I$ and therefore $r_{R / I}(M) \subseteq r(M)$ for every $M \in R_{R}^{\bmod }$. Thus $r(R)=R$.

\section{References}

[1] M.F. Atiyah, I.G. Macdonald, Introduction to commutative algebra (Addison-Wesley, Reading, Massachussetts; London; Don Mills, Ontario; 1969).

[2] Hyman Bass, "Finitistic dimension and a homological generalization of semi-primary rings", Trans. Amer. Math. Soc. 95 (1960), 466-488.

[3] Richard Courter, "Finite direct sums of complete matrix rings over perfect completely primary rings", Canad. J. Math. 21 (1969), 430-446. 
[4] Spencer E. Dickson, "A torsion theory for abelian categories", Trans. Amer. Math. Soc. 121 (1966), 223-235.

[5] Vlastimil Dlab, "Distinguished sets of ideals of a ring", Czechos Zovak Math. J. 18 .(93) (1968), 560-567.

[6] Vlastimil Dlab, "On a class of perfect rings", Canad. J. Math. 22 (1970), 822-826.

[7] B.J. Gardner, "Rings whose modules form few torsion classes", Bulz. Austral. Math. Soc. 4 (1971), 355-359.

[8] Michal Jaegermann and Jan Krempa, "Rings in which ideals are annihilators", Fund. Math. 76 (1972), 95-107.

[9] А.П. Ііншнна, Л.А. Скорняков, [A.P. Mіకina, L.A. Skornjakov], Абелевы группы н мадули [Abelian groups and modules] (Izdat. "Nauka", Moscow, 1969).

Matematicko-fyzikální fakulta, Karlova universita,

Sokolovská,

Praha,

Czechos lovakia. 